\title{
Chaotic inflation in no-scale supergravity with string inspired moduli stabilization
}

\author{
Tianjun Li ${ }^{1,2, a}$, Zhijin $\mathbf{L i}^{3}$, Dimitri V. Nanopoulos ${ }^{3,4,5}$ \\ ${ }^{1}$ State Key Laboratory of Theoretical Physics and Kavli Institute for Theoretical Physics China (KITPC), Institute of Theoretical Physics, \\ Chinese Academy of Sciences, Beijing 100190, People's Republic of China \\ ${ }^{2}$ School of Physical Electronics, University of Electronic Science and Technology of China, Chengdu 610054, People's Republic of China \\ ${ }^{3}$ George P. and Cynthia W. Mitchell Institute for Fundamental Physics and Astronomy, Texas A\&M University, College Station, TX 77843, USA \\ ${ }^{4}$ Astroparticle Physics Group, Houston Advanced Research Center (HARC), Mitchell Campus, Woodlands, TX 77381, USA \\ ${ }^{5}$ Division of Natural Sciences, Academy of Athens, 28 Panepistimiou Avenue, Athens 10679, Greece
}

Received: 24 November 2014 / Accepted: 24 January 2015 / Published online: 5 February 2015

(C) The Author(s) 2015. This article is published with open access at Springerlink.com

\begin{abstract}
The simple chaotic inflation is highly consistent with the BICEP2 experiment, and no-scale supergravity can be realized naturally in various string compactifications. Thus, we construct a chaotic inflation model in no-scale supergravity inspired from Type IIB string compactification with an anomalous $U(1)_{X}$ gauged symmetry. We introduce two moduli $T_{1}$ and $T_{2}$ which transform non-trivially under $U(1)_{X}$, and some pairs of fundamental quarks charged under the $S U(N) \times U(1)_{X}$ gauge group. The non-trivial transformations of moduli under $U(1)_{X}$ lead to a moduli-dependent Fayet-Iliopoulos (FI) term. The modulus $T_{2}$ and the real component of $T_{1}$ are stabilized by the non-perturbative effect from quark condensation and the $U(1)_{X}$ D-term. In particular, the stabilization from the anomalous $U(1)_{X}$ D-term with moduli-dependent FI term is crucial for inflation since it gives heavy mass to the real component of the modulus $T_{1}$ while keeping its axionic part light. Choosing the proper parameters, we obtain a global Minkowski vacuum where the imaginary part of $T_{1}$ has a quadratic potential for chaotic inflation.
\end{abstract}

\section{Introduction}

Inflation is a candidate to solve several problems in the standard big bang model, such as the horizon problem, flatness problem, large structure of the Universe, etc. It is getting closer to being verified based on the recent Planck and BICEP2 observations [1,2]. Both experimental results support the single field inflation with scalar spectral index $n_{\mathrm{s}}$ around 0.96. However, the Planck results provide an upper bound on the tensor-to-scalar ratio, $r \leqslant 0.11$ at $95 \%$ C.L. [1]. The simple chaotic inflation model with quadratic potential

\footnotetext{
a e-mail: tli@itp.ac.cn
}

$V=\frac{1}{2} m^{2} \phi^{2}$ [3], whose $r$ is out of this range, is disfavored. In contrast, the Starobinsky model fits with the Planck data very well [4-6]. Consequently, it was important to realize the Starobinsky model from fundamental theories, such as the supergravity (SUGRA) theory and string theory before the $\mathrm{BICEP} 2$ results.

The no-scale SUGRA [7-11], which can be realized naturally in various string compactifications [12,13], solves the cosmological constant problem at the classical level. The Starobinsky model was realized in $S U(2,1) / U(1)$ no-scale SUGRA with Wess-Zumino superpotential [14, 15]. Following this development, the SUGRA extensions of the Starobinsky model have been revived [16-21] (for more details and references, see [22]). Besides, the Starobinsky-like inflation can be fulfilled in string theory as well [23,24].

Very recently, the BICEP2 Collaboration announced the range of tensor-to-scalar ratio based on the observations of CMB B-mode polarization, $r=0.20_{-0.05}^{+0.07}$ or $r=0.16_{-0.05}^{+0.06}$ by subtracting the dust contributions. Such large $r$ significantly changes the directions of the inflation model building. The Starobinsky model is now disfavored by the BICEP2 results. Moreover, many inflation models from string theory predict small $r$ far below 0.01 and thus contradict with the BICEP2 results [25] (The exceptional models are aligned natural inflation [26], monodromy inflation [27,28] and Mflation [29,30]). Interestingly, chaotic inflation is indeed favored after the BICEP2 results, and since it has been studied extensively [31-51].

The no-scale SUGRA is equipped with a curved Kähler manifold [7-11], which leads to non-canonical kinetic terms for the fields along non-flat directions (without shift symmetry). Normally, the fields with such a kind of kinetic terms move too fast toward the minimum, and then no inflation can 
be triggered. Alternatively, through parameter tuning it is possible to get a flat direction for inflation with very small $r$, such as in the no-scale Starobinsky model $[14,15]$. In short, these potentials are either too steep or too flat to generate chaotic inflation.

For chaotic inflation, the inflaton is preferred to be the scalar with a flat direction on the Kähler manifold, while all the other fields along the rest of the directions should be properly stabilized. The flat directions of the Kähler manifold are guaranteed by the shift symmetries of the Kähler potential. The scalar potential is also flat if the shift symmetry is not broken by the superpotential. Thus, the shift symmetry was employed to construct the chaotic inflation model in no-scale SUGRA [35] (for a related study, see [42,52]). This work is based on the SUGRA extension of the Starobinsky model, the Kähler potential is of no-scale $S U(2,1) / U(1)$ type, and the inflaton is the imaginary part of the modulus, which preserves the exact shift symmetry. However, the real component of the modulus is not stabilized during inflation since the masses of the real component and inflaton are comparable around the same scale. The point is that the shift symmetry is broken by the superpotential explicitly, in consequence there is no symmetry that can prevent the inflaton from obtaining heavy mass.

Besides the shift symmetry, moduli stabilization is also needed for chaotic inflation. The moduli can be stabilized by the non-perturbative effects [53] via the KKLT mechanism in an anti-de Sitter (AdS) vacuum, which is uplifted to a metastable de Sitter (dS) vacuum by sets of anti D3-branes where supersymmetry (SUSY) is broken explicitly. Burgess, Kallosh, and Quevedo (BKQ) suggested that the uplifting of the AdS vacua with spontaneously SUSY breaking can be realized by the D-term associated with an anomalous $U(1)_{X}$ gauge symmetry [54]. Nonetheless, the non-perturbative part of the superpotentials in both KKLT and BKQ are not invariant under anomalous $U(1)_{X}$. The gauge invariant moduli stabilization was proposed in Refs. $[55,56]$ based on the non-perturbative effect of hidden gauge symmetry. As the D-term is positive semi-definite, it is useful to construct the Minkowski or dS vacuum. The effects of the D-term on moduli stabilization and dS vacua are also studied in Ref. [57,58]. Moreover, the F-term is widely used to uplift the AdS vacua. In Ref. [59], the O'Raifeartaigh model with quantum corrections is introduced to the KKLT scenario. The heavy fields are integrated out while a light field is fixed at very small value. Its F-term contributes to the vacuum energy and uplift the AdS vacua obtained from KKLT. Similarly, the Polonyi model can uplift the AdS vacua when combined with the KKLT mechanism [60-62].

The anomalous D-term with moduli-dependent FayetIliopoulos (FI) term plays a special role in inflation. The FI term depends on the real component of the moduli only, so stabilization through such kind of D-term only gives heavy mass to the real component while the imaginary or axion-like part remains light. This is different from the stabilization by the F-term or D-term with constant FI term, in which cases both the real and the imaginary components appear in the potentials and it is difficult to separate the masses between the real and imaginary parts at different scales. Instead of stabilizing the moduli directly, chaotic-like inflation can also be obtained in no-scale SUGRA by minimizing a term combining the moduli and matter fields [63-66], nevertheless, the moduli are indeed not stabilized during inflation.

In this work, we will apply the shift symmetry of the moduli to obtain chaotic inflation, where the inflaton is the corresponding axion-like field. In particular, the shift symmetry is preserved in both Kähler potential and superpotential. So it can be consistently gauged to form the anomalous $U(1)_{X}$ as long as the gauge anomaly is canceled. The Kähler potential is inspired from the Type IIB string compactification, where two moduli $T_{1}$ and $T_{2}$ are charged under the anomalous $U(1)_{X}$ gauge symmetry. One of the moduli $T_{2}$ is stabilized by the KKLT mechanism in a gauge invariant way. The real component of $T_{1}$ is automatically stabilized by the D-term associated with the anomalous $U(1)_{X}$, while its imaginary component remains light and is a natural candidate for the inflaton. We choose the moduli stabilization scale at least one order of magnitude higher than the inflation scale so that the inflation and moduli stabilization can be separated into two stages.

This paper is organized as follows. In Sect. 2, we briefly review gauge invariant moduli stabilization based on nonperturbative effects. In Sect. 3, we discuss the anomaly cancelation of the anomalous $U(1)_{X}$ symmetry. In Sect. 4, we show by combining the non-perturbative effects and the Dterm, all the moduli except the axion-like imaginary component of $T_{1}$ are stabilized. Choosing the proper parameters, we get the Minkowski vacuum and a light axion-like field with quadratic potential, which generates chaotic inflation in the scale far below the moduli stabilization scale. We discuss model building and then conclude in Sect. 5 .

\section{Gauge invariant moduli stabilization}

In the KKLT proposal, the dilaton and complex-structure moduli of Calabi-Yau compactification are fixed by the background NSNS and RR fluxes. Thus, there is only one Kähler modulus, $T$, which is not fixed by the fluxes. The SUGRA description of its low-energy effective theory is given by the Kähler potential

$K=-3 \log (T+\bar{T})$

and the superpotential

$W=W_{0}+W_{\mathrm{np}}$, 
where the constant term $W_{0}$ is obtained from the fluxes which are used to stabilize the dilaton and complex-structure moduli, and the non-perturbative term $W_{\mathrm{np}}=A \mathrm{e}^{-a T}$ is generated by the Euclidean D3-branes or alternatively by gaugino condensation within a non-Abelian sector from a stack of wrapped D7-branes. The generic F-term scalar potential is given by

$V_{\mathrm{F}}=\mathrm{e}^{K}\left(K^{i \bar{j}} D_{i} W D_{\bar{j}} \bar{W}-3 W \bar{W}\right)$,

in which $K^{i \bar{j}}$ is the inverse of the Kähler metric $K_{i \bar{j}}=$ $\partial_{i} \partial_{\bar{j}} K$. The potential of the modulus $T$ admits a supersymmetric AdS vacua where $T$ is stabilized. There are several ways to uplift the AdS vacua to $\mathrm{dS}$ vacua. In the original KKLT proposal, the AdS vacuum is uplifted by anti D3branes, which generates a non-supersymmetric term in the scalar potential,

$V=V_{\mathrm{F}}+\frac{D}{\sigma^{2}}$,

where $\sigma=\operatorname{Re}(T)$, and $D$ is a constant.

Instead of breaking SUSY explicitly, the AdS vacua can be uplifted by the D-term in the BKQ proposal [54]. In general, the D-term for the four-dimensional $N=1$ gauged SUGRA with Kähler term $G=K(\phi, \bar{\phi})+\log (W \bar{W})$ is

$V_{\mathrm{D}}=\frac{1}{2} D_{a} D^{a}$

where the gauge indices are raised by the form $\left[(\operatorname{Ref})^{-1}\right]^{a b}$ with $f$ the gauge kinetic function. The $D_{a}$ components are

$D_{a}=i K_{i} X_{a}^{i}+i \frac{W_{i}}{W} X_{a}^{i}$,

or

$D_{a}=i K_{i} X_{a}^{i}$

if $W$ is gauge invariant. Here, the $X_{a}^{i}$ are the components of the Killing vector $X_{a}=X_{a}^{i}(\phi) \partial / \partial \phi^{i}$,

In the BKQ proposal, the Killing vector has components $X^{T}=\frac{2 E}{3} i$ and $X^{Q_{i}}=i q_{i} Q_{i}$. So the D-term is

$V_{\mathrm{D}}=\frac{g_{\mathrm{YM}}^{2}}{2} D^{2}=\frac{2 \pi}{\sigma}\left(\frac{E}{\sigma}+\sum q_{i}\left|Q_{i}\right|^{2}\right)^{2}$,

where $g_{\mathrm{YM}}^{2}=4 \pi / \sigma, Q_{i}$ are the matter fields which transform linearly under the anomalous $U(1)_{X}$ with charges $q_{i}$, and the modulus $T$ shifts under the anomalous $U(1)_{X}$ and then is related to a field dependent FI term. That the D-term should be non-vanishing (non-cancellability) is a critical assumption to uplift the AdS vacua. It was argued that the matter fields $Q_{i}$ can obtain vacuum expectation value (VEV) $\left\langle Q_{i}\right\rangle=0$, so the D-term is similar to the effect of the anti D3-branes. The uplift of AdS vacua can also be done by F-term (For example, see $[67,68]$.).
As noticed in the BKQ proposal and Ref. [69,70], it is not consistent to directly add the D-term in KKLT mechanism. The modulus $T$ shifts under anomalous $U(1)_{X}: T \rightarrow$ $T+X^{T} \epsilon$, where $X^{T}$ is the Killing vector generating $U(1)_{X}$ transformation of modulus $T$. The non-perturbative term in the superpotential $W_{\mathrm{np}}$ is not gauge invariant: $W_{\mathrm{np}} \rightarrow$ $\mathrm{e}^{-a X^{T} \epsilon} W_{\mathrm{np}}$. So a field dependent coefficient in the nonperturbative term is required to cancel the factor $\mathrm{e}^{-a X^{T} \epsilon}$ if the shift symmetry can be gauged consistently.

A gauge invariant non-perturbative superpotential was constructed in Refs. [55,56]. The expression $W_{\mathrm{np}}=A \mathrm{e}^{-a T}$ in KKLT is replaced by $W_{\mathrm{np}}=F\left(Q_{i}\right) \mathrm{e}^{-a T}$, in which $F\left(Q_{i}\right)$ is a product of matter fields $Q_{i}$. The $F\left(Q_{i}\right)$ transformation under $U(1)_{X}$ cancels the phase factor $\mathrm{e}^{-a X^{T} \epsilon}$ so that the new $W_{\mathrm{np}}$ is indeed invariant under $U(1)_{X}$. Such a kind of superpotential, which originated from gaugino condensation, has been studied in Refs. [71-74]. Specifically, the model employs $N_{0}$ fundamental quark pairs, $\left(Q_{i}, \bar{Q}_{i}\right)$ under gauge group $S U(N) \times U(1)_{X}$, and the $U(1)_{X}$ charges of the quark pairs are $(q, \bar{q})$. The quarks condense and form the composite meson fields $|M|^{2}=\left|M_{i}\right|^{2}$, in which $M_{i}^{2}=Q^{i} \bar{Q}_{i}$ for $i=1, \ldots, N_{0}$. Consequently, the effective superpotential after the condensation is

$W_{\mathrm{np}}=\left(N-N_{0}\right) M^{-\frac{2 N_{0}}{\left(N-N_{0}\right)}} \mathrm{e}^{\frac{(q+\bar{q}) N_{0} T}{\left(N-N_{0}\right) \delta_{\mathrm{GS}}}}$,

in which the $i \delta_{\mathrm{GS}}=X^{T}$ and its value is determined by the quantum anomaly cancelation conditions for $S U(N)^{2} \times$ $U(1)_{X}$ and $U(1)_{X}^{3}$, which will be discussed later. Obviously, the above superpotential is gauge invariant. Opposite signs are assigned for $q+\bar{q}$ and $\delta_{\mathrm{GS}}$. The $U(1)_{X} \mathrm{D}$-term is

$V_{\mathrm{D}} \propto\left(N_{0}(q+\bar{q})|M|^{2}-\frac{3 \delta_{G S}}{2 \sigma}\right)^{2}$.

This D-term is non-vanishing for $\sigma<\infty$ and its minimum is located at $\langle M\rangle=0$. The non-cancellability assumption, which is crucial for the BKQ proposal, now is realized in the condensation mechanism. However, the non-cancellability directly results from the fact that $\delta_{\mathrm{GS}} /(q+\bar{q})<0$; by introducing more fields charged under $U(1)_{X}$, this noncancellability disappears.

The modulus $T$ and the composite field $M$ can be stabilized by minimizing $V_{\mathrm{F}}$ or the combination $V_{\mathrm{F}}+V_{\mathrm{D}}$. Based on purely $V_{\mathrm{F}}$, the vacua are of AdS as usual, uplifting from the D-term results in the $\mathrm{dS}$ vacua.

\section{Anomaly cancelation of anomalous $U(1)_{X}$}

Anomalous $U(1)_{X}$ symmetry is obtained in the heterotic string by gauging the shift symmetry of the axion-dilaton multiplet $S$ [75]. The gauge kinetic term is 


$$
\int \mathrm{d}^{2} \theta f W_{\alpha}^{2}
$$

where $W_{\alpha}$ is the field strength of the $U(1)_{X}$ vector superfield, and the gauge kinetic function is taken as $f=S$. The gauge kinetic term contains two parts $\operatorname{Re}(f) F^{2}$ and $\operatorname{Im}(f) F \tilde{F}$ [76]. The second term has a non-trivial transformation of $S$ and plays a crucial role in gauge anomaly cancelation through the Green-Schwarz mechanism in fourdimensional spacetime [77]. The quantum anomaly of $U(1)_{X}$ is canceled by the term introduced from the transformation $S \rightarrow S+i \epsilon \delta_{\mathrm{GS}}$. However, for the heterotic string case, the anomalous $U(1)_{X}$ is very constrained. As argued in Ref. [75], only the superfield $S$ can be transformed non-trivially under anomalous $U(1)_{X}$, otherwise there will be unwanted mass terms and tadpoles at tree level. The FI terms introduced by $S$ appear in the heterotic string at higher loop levels, so they are expected to be much smaller than the tree-level potential. In consequence, they are not useful if a large D-term is needed

In Type IIB string compactification, generally there are several moduli, $T_{i}$, from the Calabi-Yau space. The modulidependent part of the gauge kinetic function is $f=g_{a}^{i} T_{i}$, where $g_{a}^{i}$ are positive constants. Anomaly cancelation is determined by the component $g_{a}^{i} \operatorname{Im}\left(T_{i}\right) F_{a} \tilde{F}_{a}$. Given the moduli $T_{i}$ transform as $T_{i} \rightarrow T_{i}+i \delta_{a}^{i} \epsilon$ under $U(1)_{a}$, the anomaly cancelation requires

$\sum g_{a}^{i} \delta_{a}^{i}=-\Delta$

where $\Delta$ is the coefficient of the gauge anomaly from the fermionic contributions $\Delta F_{a} \tilde{F}_{a}$. Specifically, for the $U(1)_{X}^{3}$ gauge anomaly, the above anomaly cancelation turns into

$\sum g_{X}^{i} \delta_{X}^{i}=-\frac{1}{48 \pi^{2}} \sum q_{m}^{3}$

in which $q_{m}$ are the charges of quarks, and a factor $1 / 3$ is attributed to the over-counting of the anomaly diagrams.

\section{Chaotic inflation model building}

From the above discussions, the anomalous $U(1)_{X}$ in Type IIB string theory instead of heterotic string theory is preferred. In particular, in the Type IIA intersecting D6-brane model building or its T-dual Type IIB D3-D7 brane model building, we will not only have up to four anomalous $U$ (1) gauge symmetries, but also we have the hidden sector with additional gauge groups and exotic particles [7882]. Inspired by these string constructions, we consider the following Kähler potential:

$$
\begin{aligned}
K= & -\log \left(T_{1}+\bar{T}_{1}\right)-2 \log \left(T_{2}+\bar{T}_{2}\right) \\
& +\sum_{i=1}^{N_{0}}\left(Q_{i} \bar{Q}_{i}+\tilde{Q}_{i} \overline{\tilde{Q}}_{i}\right)+S \bar{S}-\frac{(S \bar{S})^{2}}{\Lambda_{1}^{2}} \\
& +X \bar{X}-\frac{(X \bar{X})}{\Lambda_{2}^{2}},
\end{aligned}
$$

where $T_{1}$ and $T_{2}$ transform non-trivially, $T_{i} \rightarrow T_{i}+i \delta_{\mathrm{GS}}^{i} \epsilon$ under the anomalous $U(1)_{X}$, the $N_{0}$ quark pairs $\left(Q_{i}, \tilde{Q}_{i}\right)$ form fundamental representation of $S U(N)$ gauge symmetry with $U(1)_{X}$ charges $(q, \tilde{q})$. As proposed before, the quarks $Q_{i}$ condense and form composite meson fields $M_{i}=\sqrt{Q^{i} \tilde{Q}_{i}}$. The superfields $S$ and $X$ are neutral under $S U(N) \times U(1)_{X}$, and the higher-order terms $(S \bar{S})^{2} / \Lambda_{1}^{2}$ and $(X \bar{X})^{2} / \Lambda_{2}^{2}$ from quantum corrections are needed to fix $S$ and $X$ at $\langle S\rangle=\langle X\rangle=0$ during inflation. $S$ is from the O'Raifeartaigh model and used to uplift the AdS vacua in the KKLT mechanism [59], while $X$ provides the non-vanishing F-term for inflation.

The kinetic terms of the fields $\phi^{i} \equiv\left(T_{i}, Q_{i}, \tilde{Q}_{i}, S, X\right)$ are given by $L_{\text {kin }}=K_{i j} \partial_{\mu} \phi^{i} \partial^{\mu} \bar{\phi}^{\bar{j}}$ with the Kähler metric $K_{i \bar{j}} \equiv$ $\partial^{2} K / \partial \phi^{i} \partial \bar{\phi}^{j}$. From the Kähler potential in Eq. (14), fields $Q_{i}, \tilde{Q}_{i}, S$, and $X$ (at lowest level) have canonical kinetic terms, while for the moduli $T_{i}$ with no-scale type Kähler potential, their kinetic terms are

$L_{K}=\frac{\partial_{\mu} T_{1} \partial^{\mu} \bar{T}_{1}}{\left(T_{1}+\bar{T}_{1}\right)^{2}}+\frac{2 \partial_{\mu} T_{2} \partial^{\mu} \bar{T}_{2}}{\left(T_{2}+\bar{T}_{2}\right)^{2}}$.

The gauge kinetic term consists of two parts, $S U(N)$ and $U(1)_{X}$. Here, we focus on the $U(1)_{X}$ due to quark condensations. The $S U(N)^{2} \times U(1)_{X}$ gauge anomalies are canceled by the shifts of the gauge kinetic function $f_{S U(N)} \propto g_{a} T_{2}$ under the anomalous $U(1)_{X}$. The gauge kinetic term of $U(1)_{X}$ is

$\int \mathrm{d}^{2} \theta\left(g_{1} T_{1}+g_{2} T_{2}\right) W_{\alpha}^{2}$.

The parameters $\delta_{\mathrm{GS}}^{i}, g_{1}$, and $g_{2}$ are free as along as the anomaly cancelation conditions are satisfied. Here we take

$\delta \equiv \delta_{\mathrm{GS}}^{2}=-\delta_{\mathrm{GS}}^{1}$,

and $g_{1}=1, g_{2}=2$. The anomaly cancelation condition in Eq. (13) gives

$\delta=-\frac{N N_{0}\left(q^{3}+\bar{q}^{3}\right)}{48 \pi^{2}}$.

The superpotential of the gauged SUGRA, first of all, should be gauge invariant. If there is only one modulus transforms non-trivially under anomalous $U(1)_{X}$, then the formula of the superpotential is strongly constraint by the gauge invariance. There is only one choice $W(T) \sim \mathrm{e}^{a T}$, just the effective superpotential from non-perturbative effects. However, by employing two moduli $\left(T_{1}, T_{2}\right)$ with Killing vector 
$X^{T_{1}}=i \delta_{a}, \quad X^{T_{2}}=-i \delta_{b}$,

the constraint is relaxed. The combination of the two moduli $\delta_{b} T_{1}+\delta_{a} T_{2}$ is automatically gauge invariant, and then any function in terms of $\delta_{b} T_{1}+\delta_{a} T_{2}$ is gauge invariant. This is crucial to construct the gauge invariant superpotential.

We consider the following superpotential:

$$
\begin{aligned}
W= & w_{0}+\left(N-N_{0}\right) M^{-\frac{2 N_{0}}{N-N_{0}}} \mathrm{e}^{\frac{N_{0}(q+\tilde{q}) T_{2}}{\delta\left(N-N_{0}\right)}} \\
& -\mu S+a X\left(T_{1}+T_{2}+s\right),
\end{aligned}
$$

where the first two terms $\left(W_{\mathrm{st}}\right)$ are for the gauge invariant stabilization of modulus $T_{2}$. The third term is to uplift the AdS vacuum, while the last term $\left(W_{\text {in }}\right)$ is to generate chaotic inflation, and $s$ is a constant. Because of the axionic shift symmetry of the Kähler moduli, the perturbative terms in $W_{\text {in }}$ are forbidden in the superpotential. There are two possible ways to generate $W_{\text {in }}$. First, the Kähler moduli appear in the superpotential through the non-perturbative effects, which are of the form $\propto \mathrm{e}^{-k_{i} T_{i}}=1-k_{i} T_{i}+\cdots$ (higher - order terms) for small $k_{i}$. Assuming the coefficients satisfy $k_{1}=k_{2} \ll 1$, we simply consider the constant and linear terms. As long as the exponential term is coupled with the matter field $X$, we get the inflation term $W_{\text {in }}$ in (20). Therefore, the superpotential $W_{\text {in }}$, which realizes chaotic inflation after moduli stabilization, can be considered as small $k_{i} \ll 1$ approximation of the typical non-perturbative effect. The superpotential $\propto \mathrm{e}^{-a T}$ is employed to realize natural inflation in the minimal supergravity [83-85]. Actually this small $k_{i}$ approximation corresponds to the well-known relationship that with large axion decay constant (small $k_{i}$ in our model), the natural inflation gets close to the chaotic inflation. Second, we can consider the flux compactifications, and there exist Kähler moduli in the superpotential. For example, see [82] and references therein.

For the moduli stabilization, we require that $W_{\text {st }}$ be "hierarchically" larger than the term $W_{\text {in }}$. To be concrete, we will take $w_{0} \simeq 2.0 \times 10^{-3}$ in Planck units, while the parameter $a$, which corresponds to the inflaton mass, is about $10^{13} \mathrm{GeV}$, or $\sim 10^{-5}$ in Planck units. Therefore, the term $W_{\text {in }}$ has ignorable effect on the moduli stabilization. Conversely, once the moduli are fixed, they are completely frozen out during inflation.

Given $T_{2} \equiv \phi+i \theta, M \equiv m \mathrm{e}^{i \beta}$, and $a=-\frac{N_{0}(q+\tilde{q})}{\delta\left(N-N_{0}\right)}$, $b=\frac{2 N_{0}}{N-N_{0}}$, the F-term potential is

$$
\begin{aligned}
V_{\mathrm{F}}= & \frac{\mathrm{e}^{2 N_{0} m^{2}}}{4\left(T_{1}+\bar{T}_{1}\right) \phi^{2}}\left\{\mathrm { e } ^ { - 2 a \phi } \left[4\left(N-N_{0}\right)^{2} m^{-2 b}\right.\right. \\
& \times\left(\frac{1}{2} a^{2} \phi^{2}+a \phi\right)+\frac{\left(N-N_{0}\right)^{2}}{2 N_{0}} b^{2} m^{-2(b+1)} \\
& \left.-2 b\left(N-N_{0}\right)^{2} m^{-2 b}+2 N_{0} m^{2-2 b}\left(N-N_{0}\right)^{2}\right] \\
& +2 N_{0} m^{2} w_{0}^{2}+2 w_{0}\left(N-N_{0}\right) m^{-b} \mathrm{e}^{-a \phi}
\end{aligned}
$$

$$
\begin{aligned}
& \times\left(2 a \phi+2 N_{0} m^{2}-b\right) \cos (a \theta+b \beta) \\
& \left.+\mu^{2}+a^{2}\left|T_{1}+T_{2}+s\right|^{2}+\cdots\right\},
\end{aligned}
$$

in which the terms proportional to $S$ and $X$ are ignored. The potential depends on the combination $a \theta+b \beta$. From the potential (21), $\phi$ and $m$ obtain non-zero VEVs. As both $T_{2}$ and $M$ are charged under anomalous $U(1)_{X}$, the $U(1)_{X}$ gauge symmetry is spontaneously broken. For the axion $\theta$ and the phase $\beta$, one of their linear combination is absorbed by the massive $U(1)_{X}$ gauge field and becomes its longitudinal component through the combined effect of the Stückelberg and Higgs mechanisms, while another degree of freedom is strongly stabilized by the potential (21).

From the potential (21), the term $\propto a^{2}$ is several orders smaller than the KKLT terms and only has small correction to the moduli stabilization. Its effect will be studied later. The modulus $T_{2}$ and meson $M$ are stabilized by the KKLT term, which gives an AdS vacuum. The AdS vacuum is raised by the term $\propto \mu^{2}$. In our model, taking $N=10$ with only one flavor of quarks, $q+\tilde{q}=-4 \pi \delta$, the constant $w_{0}=$ 0.002 , the vacuum locates at $\phi_{0}=8.2990, m_{0}=0.0879$, and $a \theta+b \beta=(2 n+1) \pi$. To uplift the AdS vacuum to a Minkowski vacuum, we require $\mu^{2}=6.58 \times 10^{-6}$. The masses of the fields $\phi$ and $m$ (after canonical normalization) are $m_{\phi}=1.2 \times 10^{-3}$ and $m_{m}=1.3 \times 10^{-4}$. Note that the off-diagonal entry is much smaller and the Hessian matrix is positive definite, we confirm that above vacuum is real. The Kähler modulus $T_{2}$ is stabilized in the region $\operatorname{Re}\left(\mathrm{T}_{2}\right) \simeq$ $8.3>1$ and even larger at the order 10 by taking larger $N$, so the supergravity approximation is valid. However, the moduli cannot be stabilized at much larger value. Otherwise, the moduli stabilization is not consistent with the inflation energy scale. For the two axionic fields $\theta$ and $\beta$, the remaining degree of freedom after symmetry broken is stabilized by the vacuum condition $a \theta+b \beta=(2 n+1) \pi$. As an example, taking the $U(1)_{X}$ gauge $\theta=0$, the phase $\beta$ is stabilized at $(2 n+1) \pi / b$ with mass $m_{\beta}^{2}=6.2 \times 10^{-4}$.

The Hubble scale is around $5 \times 10^{-5}$ for $r \simeq 0.16$. Thus, the fields $\phi, m$, and the remaining axionic field after the $U(1)_{X}$ symmetry breaking can be stabilized during inflation. Besides, the gravitino mass is $4.5 \times 10^{-5}$, which will not affect the inflation in no-scale supergravity. Moreover, we present the potential for moduli stabilization in Fig. 1.

The D-term associated with the anomalous $U(1)_{X}$ is

$$
\begin{aligned}
V_{\mathrm{D}}= & \frac{1}{2 \operatorname{Re}\left(T_{1}+2 T_{2}\right)} \\
& \times\left(\frac{\delta}{T_{1}+\bar{T}_{1}}-\frac{2 \delta}{T_{2}+\bar{T}_{2}}+N_{f}(q+\tilde{q})|M|^{2}\right)^{2} .
\end{aligned}
$$

The D-term for another gauge group $S U(N)$ has already vanished under the quark condensation $\left|Q_{i}\right|^{2}=\left|\tilde{Q}_{i}\right|^{2}$. In Eq. (22) the $\delta$ has opposite sign to the charge $q+\tilde{q}$, the 


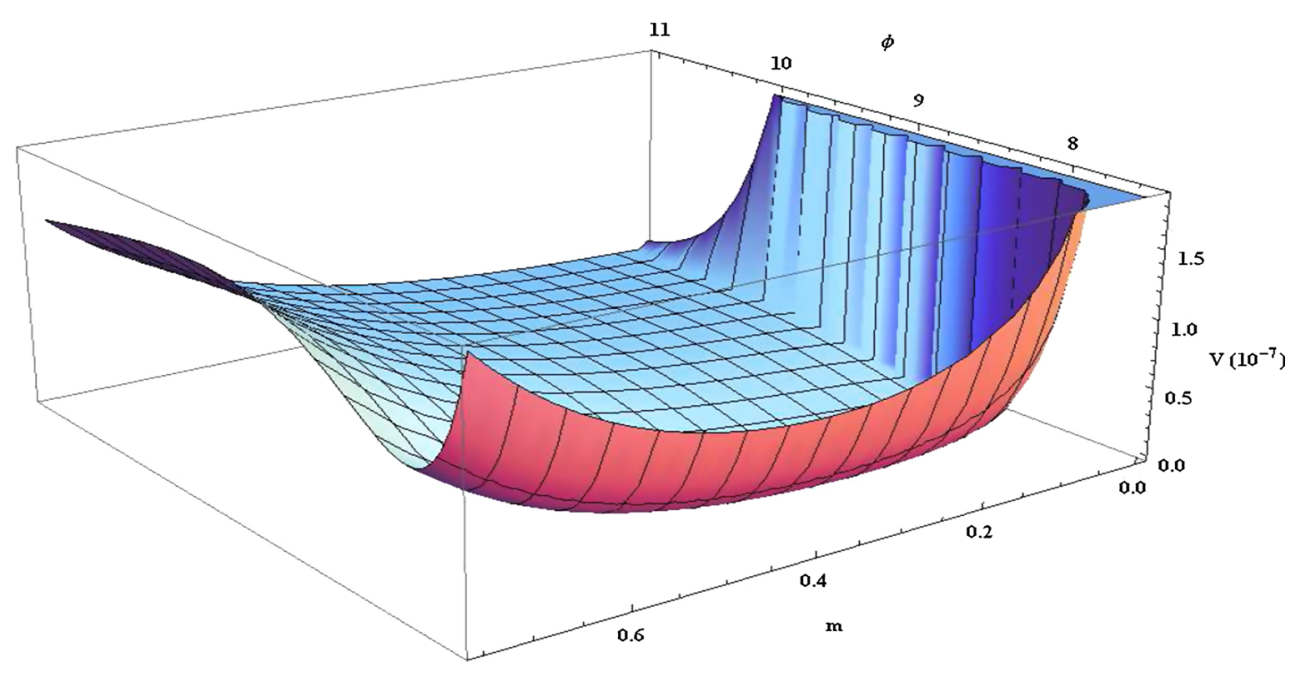

Fig. 1 F-term moduli stabilization in a Minkowski vacuum

above D-term is canceled by shifting the real component of modulus $T_{1}$ for any given $T_{2}$ and $M$. This is completely different from the case with modulus $T_{2}$ only, in which the $|M|^{2}$ has the same sign with the modulus-dependent FI term and non-cancellability is guaranteed. At the vacuum, the D-term vanishes, and gives a large mass to the field $\operatorname{Re}\left(T_{1}\right)$. So even though we can gauge invariantly fix the modulus $T_{2}$ and $M$, the D-term uplifting of the AdS vacua is not feasible.

The modulus $T_{2}$ and $M$ are fixed at $\left\langle T_{2}\right\rangle=\phi_{0}+i \theta_{0}$ and $|M|=m_{0}$. For simplicity, we will take the $U(1)_{X}$ gauge $\theta_{0}=0$. The real component of modulus $T_{1}$ obtains a large mass and is stabilized as well. For $T_{1}=\sigma+i \rho$, the vacuum locates at

$\sigma_{0}=\frac{1}{2}\left(\frac{1}{\phi_{0}}+\left|\frac{q+\tilde{q}}{\delta}\right| N_{0} m_{0}^{2}\right)^{-1}$

with mass (before rescaling)

$m_{\sigma}^{2}=\left.\frac{\partial^{2} V_{\mathrm{D}}}{\partial \sigma^{2}}\right|_{\sigma_{0},\left\langle T_{2}\right\rangle, m_{0}}=\frac{8 \delta^{2}}{\phi_{0}^{5}} \frac{\left(1-N_{0}(q+\tilde{q}) \phi_{0} m_{0}^{2} / \delta\right)^{5}}{5-4 N_{0}(q+\tilde{q}) \phi_{0} m_{0}^{2} / \delta}$.

With parameters given before, $m_{0}^{2} \ll 1$, we have $\sigma_{0} \simeq \phi_{0} / 2$ and $m_{\sigma}^{2} \simeq 8 \delta^{2} / 5 \phi_{0}^{5}$. The physical mass of $\sigma$ (after rescaling) is about $2 \delta / \sqrt{5} \phi_{0}^{1.5}$, above $10^{16} \mathrm{GeV}$ providing $\delta \sim$ $O\left(10^{-1}\right)$. It is much larger than the Hubble scale, and then the real component of modulus $T_{1}$ is strongly stabilized during inflation.

Here the moduli-dependent FI term plays a crucial role in the moduli stabilization, as it is independent with the imaginary components of the moduli, we can safely stabilize the real component while keep the axion-like imaginary component light.

Figure 2 shows the D-term potential with stabilized $T_{2}$, where the quark condensation term is ignored. The field $\sigma$ has

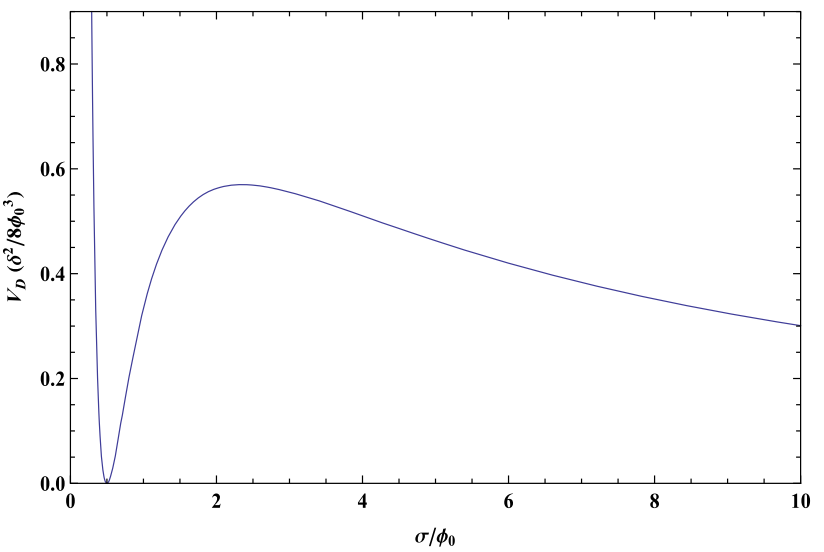

Fig. 2 Anomalous $U(1)_{X}$ D-term with unit $\frac{\delta^{2}}{8 \phi_{0}^{3}}$. The $x$ axis is scaled by $\phi_{0}$, and the quark condensation term is ignored. With non-vanished $m_{0}^{2}$ term the $\langle\sigma\rangle$ will shift to the left and makes the minimum valley steeper

a steep minimum at $\phi_{0} / 2$, which is also the global minimum as a result of the cancellability. Besides, the potential shows run away tendency corresponding to the decompactification.

During inflation, the fields $\phi, m, \theta$, and $\beta$ in (21) are frozen out since one linear combination of them is absorbed by heavy massive vector field and all the rest obtain large masses. The potential is dominated by the inflation term $a^{2}\left|T_{1}+T_{2}+s\right|^{2}$ as

$\left.V_{\mathrm{F}}\right|_{\phi_{0}, m_{0}}=\frac{a^{2}}{8 \phi_{0}^{2} \sigma}\left(\rho^{2}+\left(\sigma+\phi_{0}+s\right)^{2}\right)$,

where the F-term coefficient $\mathrm{e}^{2 N_{0} m_{0}^{2}} \simeq 1$ is ignored and the $U(1)_{X}$ gauge $\theta=0$ is applied. The field $\sigma$ is fixed at $\sigma=\sigma_{0}$ by the D-term flat condition. To get a Minkowski vacuum one requires $s=-\left(\sigma_{0}+\phi_{0}\right)$. During inflation the inflaton $\rho$ is large, the inflationary potential couples with $\sigma$ and thus 
gives a small correction to the $\sigma$ stabilization. The overall potential near the vacuum is

$$
\begin{aligned}
V & =\frac{a^{2}}{8 \phi_{0}^{2} \sigma}\left(\rho^{2}+\left(\sigma-\sigma_{0}\right)^{2}\right)+V_{\mathrm{D}} \\
& \simeq V_{\mathrm{I}} \frac{\sigma_{0}}{\sigma}+\frac{1}{2} m_{\sigma}^{2}\left(\sigma-\sigma_{0}\right)^{2},
\end{aligned}
$$

where $V_{\mathrm{I}}=\frac{a^{2}}{8 \phi_{0}^{2} \sigma_{0}} \rho^{2}$ is the inflaton potential. The VEV of $\sigma$ is shifted to $\sigma \simeq \sigma_{0}\left(1+V_{I} / m_{\sigma}^{2} \sigma_{0}^{2}\right)$. Consequently, the inflationary potential will be slightly reduced by $V_{\mathrm{I}}^{2} / 2 m_{\sigma}^{2} \sigma_{0}^{2}$. Since $V_{\mathrm{I}} \sim 10^{-8} \ll m_{\sigma}^{2} \sigma_{0}^{2}$, this shift is ignorable, and so is its effect on the inflaton potential.

From Eq. (26), we get the scalar potential for the only unstabilized scalar $\rho$

$V_{\text {in }}(\rho)=\frac{a^{2}}{8 \sigma_{0} \phi_{0}^{2}} \rho^{2}$.

And its kinetic term in Eq. (15) is

$L_{K}=\frac{1}{4 \sigma_{0}^{2}} \partial_{\mu} \rho \partial^{\mu} \rho$.

Redefining the field $\psi \equiv \rho / \sqrt{2} \sigma_{0}$, the Lagrangian for the canonically normalized field $\psi$ is

$L=\frac{1}{2} \partial_{\mu} \psi \partial^{\mu} \psi+\frac{\sigma_{0}}{4 \phi_{0}^{2}} a^{2} \psi^{2}$.

Chaotic inflation can be driven by a scalar $\psi$ with a quadratic potential, which is well known to be consistent with the BICEP2 observations, especially for the large tensor-toscalar ratio $r \simeq \frac{8}{N_{\mathrm{e}}}$, where $N_{\mathrm{e}}$ is the e-folding number of the universe scale expansion during inflation process. To be consistent with the observations, the inflaton mass is about $m_{\psi} \simeq 1.8 \times 10^{13} \mathrm{GeV}$. Therefore, the parameter $a=\left(2 / \sigma_{0}\right)^{1 / 2} \phi_{0} m_{\psi} \sim 10^{-5}$ in Planck unit, as discussed before.

\section{Discussions and conclusion}

In this work we have constructed the chaotic inflation model in the no-scale SUGRA inspired from Type IIB string compactification. The inflation models in no-scale SUGRA generically give a small tensor-to-scalar ratio $r<0.01$ $[14,15,20-22]$, which are strongly disfavored by the recent BICEP2 observations [2]. For a lot of stringy inflation models, they are realized from the string low-energy effective actions, which are of no-scale type and obtain small $r$ as well [25]. The inflationary models with small $r$ are driven by the scalars which are non-flat directions on the Kähler manifold. The potentials of the these scalar fields are either too steep for inflation, or of plateau type with small $r$ after tuning. Therefore, as correctly noticed in [35], it is necessary to employ the fields which are flat directions of Kähler manifold. The Kähler potential is invariant under the shift of such fields.

However, having only shift symmetry does not guarantee inflation. The extra moduli except the inflaton should be frozen during inflation to generate single field inflation. The moduli can be stabilized by non-perturbative effects like the KKLT mechanism. However, once the extra moduli are stabilized, the inflaton, which has shift symmetry, also obtains mass at the same scale and then destroys the inflation $[35,83]$. In short, the inflaton with shift symmetry in the Kähler potential does not have a light mass as expected. The point is that the shift symmetry provided in the Kähler potential $K$ is broken by the superpotential $W$ explicitly. To obtain a light modulus, the shift symmetry should be kept in the whole Kähler function $G=K+\log (W \bar{W})$.

If there is just one modulus $T$ and an anomalous $U(1)_{X}$ gauge symmetry, the only modulus-dependent superpotential, which is invariant up to a phase factor under shift transformation, is $\mathrm{e}^{-a T}$. So this is just the effective superpotential from the non-perturbative effects. However, it is impossible to get a quadratic potential for the chaotic inflation with such a superpotential. In this work, we have solved this problem by using two moduli that transform non-trivially under the $U(1)_{X}$, so that we can construct a polynomial gauge invariant superpotential. In string theory, the Kähler moduli admit shift symmetry which prohibits such kind of polynomial terms in the superpotential. However, the perturbative term in our model can be obtained from the non-perturbative effect $X \mathrm{e}^{-k\left(T_{1}+T_{2}\right)}$ under small $k \ll 1$ approximation, in which the higher-order terms proportional to $k^{n}(n \geqslant 2)$ are ignored. This approximation is similar to the relationship between the natural inflation and the chaotic inflation: with increasing axion decay constant, the natural inflation gets close to the chaotic inflation. The alternative way to generate such term is to consider flux compactifications.

The KKLT proposal also needs to be modified for the anomalous $U(1)_{X}$, as in the initial case the non-perturbative superpotential is not invariant under the anomalous $U(1)_{X}$ [53]. This is solved by introducing a hidden gauge sector $S U(N)$ gauge group [55,56]. The non-perturbative superpotential obtained from the quark condensation is invariant under $S U(N) \times U(1)_{X}$, and leads to the moduli stabilization. It also solves the non-cancellability assumption in the BKQ proposal [54]. The moduli stabilization in our work follows this gauge invariant method, but with a different role for the D-term to play.

In our model we have considered two moduli $T_{i}$ transforming non-trivially under anomalous $U(1)_{X}$. It could be obtained from Type IIB string compactification instead of the heterotic string compactification since in the later case only the dilaton superfield can be gauged under anomalous $U(1)_{X}$ [75]. Besides, the Type IIB string compactification 
is also preferred as it allows FI term at tree level, the large D-term is needed to stabilize the moduli at string scale. We have stabilized one of the moduli $T_{2}$ by the gauge invariant non-perturbative superpotential. However, differently from Ref. [56], our D-term vanishes at the vacuum.

The cancelation of the D-term fixes the real component of another modulus $T_{1}$, whose mass can be at least one order of magnitude larger than the Hubble scale by changing the $U(1)_{X}$ charges. Nonetheless, for the axion-like component of $T_{1}$, its mass is not affected by the D-term, and keeps light after the moduli stabilization. While for the stabilization determined by the F-term or D-term with constant FI term, it is very difficult to get a light mass after stabilization as all the components interact with each other.

Acknowledgments We would like to thank the anonymous referee very much for the careful review and valuable suggestions. Z.L would like to thank Ergin Sezgin for valuable discussion. The work of DVN was supported in part by the DOE Grant DE-FG03-95-ER-40917. The work of TL is supported in part by the Natural Science Foundation of China under Grant numbers 10821504, 11075194, 11135003, and 11275246, and by the National Basic Research Program of China (973 Program) under Grant number 2010CB833000 (TL).

Open Access This article is distributed under the terms of the Creative Commons Attribution License which permits any use, distribution, and reproduction in any medium, provided the original author(s) and the source are credited.

Funded by $\mathrm{SCOAP}^{3}$ / License Version CC BY 4.0.

\section{References}

1. P.A.R. Ade et al., Planck Collaboration. arXiv:1303.5082 [astroph.CO]

2. P.A.R. Ade et al., BICEP2 Collaboration. arXiv:1403.3985 [astroph.CO]

3. A.D. Linde, Phys. Lett. B 129, 177 (1983)

4. A.A. Starobinsky, Phys. Lett. B 91, 99 (1980)

5. V.F. Mukhanov, G.V. Chibisov, JETP Lett. 33, 532 (1981) [Pisma Zh. Eksp. Teor. Fiz. 33, 549 (1981)]

6. A.A. Starobinsky, Sov. Astron. Lett. 9, 302 (1983)

7. E. Cremmer, S. Ferrara, C. Kounnas, D.V. Nanopoulos, Phys. Lett. B 133, 61 (1983)

8. J.R. Ellis, A.B. Lahanas, D.V. Nanopoulos, K. Tamvakis, Phys. Lett. B 134, 429 (1984)

9. J.R. Ellis, C. Kounnas, D.V. Nanopoulos, Nucl. Phys. B 241, 406 (1984)

10. J.R. Ellis, C. Kounnas, D.V. Nanopoulos, Nucl. Phys. B 247, 373 (1984)

11. A.B. Lahanas, D.V. Nanopoulos, Phys. Rep. 145, 1 (1987)

12. E. Witten, Phys. Lett. B 155, 151 (1985)

13. T. Li, J.L. Lopez, D.V. Nanopoulos, Phys. Rev. D 56, 2602 (1997). hep-ph/9704247

14. J. Ellis, D.V. Nanopoulos, K.A. Olive, Phys. Rev. Lett. 111, 111301 (2013). arXiv:1305.1247 [hep-th]

15. J. Ellis, D.V. Nanopoulos, K.A. Olive, JCAP 1310, 009 (2013). arXiv: 1307.3537

16. S. Cecotti, Phys. Lett. B 190, 86 (1987)

17. S. Cecotti, S. Ferrara, M. Porrati, S. Sabharwal, Nucl. Phys. B 306, $160(1988)$
18. S.V. Ketov, A.A. Starobinsky, Phys. Rev. D 83, 063512 (2011). arXiv: 1011.0240 [hep-th]

19. S.V. Ketov, A.A. Starobinsky, JCAP 1208, 022 (2012). arXiv:1203.0805 [hep-th]

20. R. Kallosh, A. Linde, JCAP 1306, 028 (2013)

21. S. Ferrara, R. Kallosh, A. Linde, M. Porrati, Phys. Rev. D 88, 085038 (2013). arXiv:1307.7696 [hep-th]

22. A. Linde. arXiv: 1402.0526 [hep-th]

23. M. Cicoli, S. Downes, B. Dutta, JCAP 1312, 007 (2013). arXiv:1309.3412 [hep-th]

24. J. Ellis, N.E. Mavromatos, D.V. Nanopoulos. arXiv:1402.5075 [hep-th]

25. C.P. Burgess, M. Cicoli, F. Quevedo, JCAP 1311, 003 (2013). arXiv:1306.3512 [hep-th]

26. J.E. Kim, H.P. Nilles, M. Peloso, JCAP 0501, 005 (2005). hep-ph/0409138

27. E. Silverstein, A. Westphal, Phys. Rev. D 78, 106003 (2008). arXiv:0803.3085 [hep-th]

28. L. McAllister, E. Silverstein, A. Westphal, Phys. Rev. D 82, 046003 (2010). arXiv:0808.0706 [hep-th]

29. A. Ashoorioon, H. Firouzjahi, M.M. Sheikh-Jabbari, JCAP 0906, 018 (2009). arXiv:0903.1481 [hep-th]

30. A. Ashoorioon, M.M. Sheikh-Jabbari, JCAP 1106, 014 (2011) arXiv:1101.0048 [hep-th]

31. K. Nakayama, F. Takahashi. arXiv:1403.4132 [hep-ph]

32. K. Harigaya, M. Ibe, K. Schmitz, T.T. Yanagida. arXiv:1403.4536 [hep-ph]

33. K. Harigaya, T.T. Yanagida. arXiv:1403.4729 [hep-ph]

34. Y. Hamada, H. Kawai, K.-y. Oda, S.C. Park. arXiv: 1403.5043 [hep$\mathrm{ph}]$

35. S. Ferrara, A. Kehagias, A. Riotto. arXiv:1403.5531 [hep-th]

36. H.M. Lee. arXiv:1403.5602 [hep-ph]

37. Y. Gong. arXiv: 1403.5716 [gr-qc]

38. L.E. Ibanez, I. Valenzuela. arXiv:1403.6081 [hep-ph]

39. A. Ashoorioon, K. Dimopoulos, M.M. Sheikh-Jabbari, G. Shiu. arXiv:1403.6099 [hep-th]

40. N. Okada, V.N. Enouz, Q. Shafi. arXiv:1403.6403 [hep-ph]

41. R. Kallosh, A. Linde, B. Vercnocke, W. Chemissany. arXiv:1403.7189 [hep-th]

42. J. Ellis, M.A.G. Garcia, D.V. Nanopoulos, K.A. Olive. arXiv: 1403.7518 [hep-ph]

43. P. Di Bari, S.F. King, C. Luhn, A. Merle, A. Schmidt-May. arXiv: 1404.0009 [hep-ph]

44. P. Creminelli, D. Lp. Nacir, M. Simonovi, G. Trevisan, M. Zaldarriaga. arXiv:1404.1065 [astro-ph.CO]

45. S. Kawai, N. Okada. arXiv:1404.1450 [hep-ph]

46. I. Oda, T. Tomoyose. arXiv:1404.1538 [hep-ph]

47. N. Kaloper, A. Lawrence. arXiv:1404.2912 [hep-th]

48. A. Hebecker, S.C. Kraus, L.T. Witkowski. arXiv:1404.3711 [hepth]

49. H. Murayama, K. Nakayama, F. Takahashi, T.T. Yanagida. arXiv:1404.3857 [hep-ph]

50. F. Farakos, R. von Unge. arXiv:1404.3739 [hep-th]

51. X. Gao, T. Li, P. Shukla. arXiv: 1404.5230 [hep-ph]

52. K. Hamaguchi, T. Moroi, T. Terada, Phys. Lett. B 733C, 305-308 (2014). arXiv:1403.7521 [hep-ph]

53. S. Kachru, R. Kallosh, A.D. Linde, S.P. Trivedi, Phys. Rev. D 68, 046005 (2003). arXiv:hep-th/0301240

54. C.P. Burgess, R. Kallosh, F. Quevedo, JHEP 0310, 056 (2003). arXiv:hep-th/0309187

55. E. Dudas, S.K. Vempati, Nucl. Phys. B 727, 139 (2005). arXiv:hep-th/0506172

56. A. Achucarro, B. de Carlos, J.A. Casas, L. Doplicher, JHEP 0606, 014 (2006). arXiv:hep-th/0601190

57. P. Binetruy, G. Dvali, R. Kallosh, A. Van Proeyen, Class. Quantum Gravity 21, 3137 (2004). arXiv:hep-th/0402046 
58. G. Villadoro, F. Zwirner, Phys. Rev. Lett. 95, 231602 (2005). arXiv:hep-th/0508167

59. R. Kallosh, A.D. Linde, JHEP 0702, 002 (2007). arXiv:hep-th/0611183

60. H. Abe, T. Higaki, T. Kobayashi, Y. Omura, Phys. Rev. D 75, 025019 (2007). arXiv:hep-th/0611024

61. H. Abe, T. Higaki, T. Kobayashi, Phys. Rev. D 76, 105003 (2007). arXiv:0707.2671 [hep-th]

62. M. Badziak, M. Olechowski, JCAP 1002, 026 (2010). arXiv:0911.1213 [hep-th]

63. K. Enqvist, D.V. Nanopoulos, M. Quiros, Phys. Lett. B 159, 249 (1985)

64. J.R. Ellis, Z. Lalak, S. Pokorski, K. Turzynski, JCAP 0610, 005 (2006). arXiv:hep-th/0606133

65. S. Antusch, M. Bastero-Gil, K. Dutta, S.F. King, P.M. Kostka, Phys. Lett. B 679, 428 (2009). arXiv:0905.0905 [hep-th]

66. T. Li, Z. Li, D.V. Nanopoulos. arXiv:1310.3331 [hep-ph]

67. O. Lebedev, H.P. Nilles, M. Ratz, Phys. Lett. B 636, 126 (2006). arXiv:hep-th/0603047

68. E. Dudas, C. Papineau, S. Pokorski, JHEP 0702, 028 (2007). arXiv:hep-th/0610297

69. K. Choi, A. Falkowski, H.P. Nilles, M. Olechowski, Nucl. Phys. B 718, 113 (2005). arXiv:hep-th/0503216

70. S.P. de Alwis, Phys. Lett. B 626, 223 (2005). arXiv:hep-th/0506266
71. T.R. Taylor, G. Veneziano, S. Yankielowicz, Nucl. Phys. B 218, 493 (1983)

72. I. Affleck, M. Dine, N. Seiberg, Nucl. Phys. B 241, 493 (1984)

73. D. Lust, T.R. Taylor, Phys. Lett. B 253, 335 (1991)

74. B. de Carlos, J.A. Casas, C. Munoz, Phys. Lett. B 263, 248 (1991)

75. M. Dine, N. Seiberg, E. Witten, Nucl. Phys. B 289, 589 (1987)

76. J. Wess, J. Bagger (Univ. Press, Princeton, 1992), p. P259

77. M.B. Green, J.H. Schwarz, Phys. Lett. B 149, 117 (1984)

78. M. Cvetic, G. Shiu, A.M. Uranga, Phys. Rev. Lett. 87, 201801 (2001). arXiv:hep-th/0107143

79. M. Cvetic, G. Shiu, A.M. Uranga, Nucl. Phys. B 615, 3 (2001). arXiv:hep-th/0107166

80. M. Cvetic, T. Li, T. Liu, Nucl. Phys. B 698, 163 (2004). arXiv:hep-th/0403061

81. C.-M. Chen, T. Li, D.V. Nanopoulos, Nucl. Phys. B 740, 79 (2006). arXiv:hep-th/0601064

82. C.-M. Chen, T. Li, Y. Liu, D.V. Nanopoulos, Phys. Lett. B 668, 63 (2008). arXiv:0711.2679 [hep-th]

83. R. Kallosh, Lect. Notes Phys. 738, 119 (2008). arXiv:hep-th/0702059 [HEP-TH]

84. M. Czerny, T. Higaki, F. Takahashi, JHEP 1405, 144 (2014). arXiv:1403.0410 [hep-ph]

85. R. Kallosh, A. Linde, B. Vercnocke, Phys. Rev. D 90, 041303 (2014). arXiv:1404.6244 [hep-th] 